\title{
U.S. National Football League Athletes Seeking Unproven Stem Cell Treatments
}

\author{
Kirstin R.W. Matthews* and Maude L. Cuchiara
}

\section{ABSTRACT}

From professionals to weekend warriors, many athletes seek unproven stem cell (SC) treatments in an effort to heal injuries nonsurgically and/or to accelerate recovery times after surgery. Among the elite athletes opting for these treatments are high-profile U.S. National Football League (NFL) players. Over the past 5 years, several NFL players have publicly advocated for SC types of treatments and credit them as a major reason they could continue their careers after injuries. In this article, we describe the current problems associated with unproven SC treatments, focusing on treatments without U.S. Food and Drug Administration approval undertaken by NFL players in the past 5 years. Specifically, we highlight the types of treatments obtained and how the clinics advertise specifically to athletes. We also review the intended and unintended consequences of high-profile players receiving and advocating for these types of therapies. Our findings suggest that NFL players increasingly seek out unproven SC therapies to help accelerate recoveries from injuries. While most seem to receive treatment within the United States, several have traveled abroad for therapies unavailable domestically.

\section{INTRODUCTION}

Each year, more than 700 stem cell (SC) clinics around the world open their doors to "stem cell tourists" [1]. Tens of thousands of patients travel abroad to seek treatment for ailments-ranging from autism to multiple sclerosis and paralysis - for which no cure exists and treatment options are limited $[2,101]$. The majority of patients journey from industrialized countries to developing

Baker Institute for Public Policy, Rice University Houston, Texas.

This material was presented at the 2014 International Society for Stem Cell Research Annual Conference.

*(Correspondence: krwm@rice.edu) ones where federal regulations may be more lenient or nonexistent. These therapies cost patients and their families on average $\$ 40,000$, not including travel expenses or additional medical costs required after the treatment $[3,4]$. Many scientists object to these treatments and advocate for empirically demonstrated clinical benefit, which has not been shown, before the therapies experience widespread use [102]. More troublesome have been the reported severe side effects of the treatments, including one death and a near death in a clinic in Germany [103].

The use of SCs as orthopedic therapies in the United States is becoming more commonplace and has drawn the attention of elite athletes [2]. In the United States, few athletes are more prominent than those from the U.S. National Football League (NFL). Recently, NFL players have been vocal about receiving $\mathrm{SC}$ treatments and their successful recoveries. Some medical treatments, such as the use of anabolic steroids and human growth hormone, were banned after being thoroughly studied by the league [5,104]. Not only were they deemed a health risk, but they were also considered a form of doping. SC treatments are relatively new and have not been reviewed in a similar manner [2]. Consequently, on the condition that SC treatments do not use a banned substance, they are not perceived as "doping," and the league has permitted athletes to receive the treatments regardless of potential safety concerns. 
In this work, we review the therapies that NFL players have received and describe how clinics target their advertising to high-performance athletes. We found that NFL players have been resorting to unproven SC therapies more often to treat orthopedic injuries. In turn, clinics have been using these players to advertise their procedures by citing this as a common practice of the NFL. We also describe how the use of SC therapies and their promotion by these prominent public figures can have effects on the public's perception on the safety and efficacy of these procedures.

\section{NFL and unproven}

\section{SC treatments in the United States}

To determine the extent of NFL player participation in unproven SC treatments, we conducted an online search of clinics, NFL players, news articles, and blogs. We found 12 NFL football players described publicly as having obtained an unproven SC treatment since 2009 (Table 1). Several players sought out clinics overseas, but others were able to receive therapies in the United States. Procedures taking place in the United States are described by the clinics as exempt from U.S. Food and Drug Administration (FDA) regulation. The FDA surgical exemption clause states "[clinics] are not required to comply with the [FDA] requirements ... if [they] are an establishment that removes [human cells, tissues, and cellular and tissue-based products (HCT/Ps)] from an individual and implants such as HCT/Ps into the same individual during the same surgical procedure" [105]. Though each clinic has proprietary methods for cell harvesting and isolation, the basic idea remains the same. Typically, adipose tissue or bone marrow is harvested from a patient in the morning. The same day, "SCs" are isolated from the tissue and implanted back into the same patient at the injury site. Because the treatments are same-day procedures involving minimal manipulation of autologous cells, they fall within the FDA surgery exemption clause. However, as a result of minimal manipulation and processing, it is also unclear which types of cells the "SC" treatment actually includes.

Previously, several clinics, including CellTex and Regenexx, also performed treatments utilizing cultured cells, but they were shut down by the FDA, as this process was deemed significant manipulation [6]. In these types of therapies, SCs harvested from the bone marrow of a patient were grown for several weeks in a laboratory before they were implanted back into a patient. The cells that are implanted are not well characterized, and it is unclear how many, if any, are truly SCs after culture. The FDA considers these cells a "biological drug product" that requires FDA approval before use in patients. The FDA filed a lawsuit against Regenexx in 2010, challenging the clinic's assertion that the cells are not significantly modified during the culture period. Regenexx and its

\section{Table 1. NFL Players Receiving Stem Cell Therapies}

\begin{tabular}{|c|c|c|c|c|c|}
\hline NFL player & Team & Position & Year & Location (clinic) & Purpose \\
\hline Bradshaw, Ahmad & New York Giants & Running Back & 2012 & Unspecified (?) & Foot \\
\hline Curry, Aaron & Oakland Raiders & Linebacker & 2012 & Unspecified (?) & Knees \\
\hline Green, Jarvis & Denver Broncos & Defensive Lineman & 2009 & USA (Regenexx) & Knee \\
\hline Landry, LaRon & Washington Redskins & Safety & 2012 & USA (?) & Achilles tendon \\
\hline Lewis, Ray & Baltimore Ravens & Linebacker & 2012 & Europe (?) & Triceps \\
\hline Manning, Peyton & Indianapolis Colts & Quarterback & 2011 & Germany (?) & Neck \\
\hline Maze, Marquis & Pittsburgh Steelers & Receiver & 2012 & USA (Precision StemCell) & Joints \& muscles \\
\hline McClain, Rolando & Oakland Raiders & Linebacker & 2012 & USA (Precision StemCell) & Knee \& ankle \\
\hline Moreno, Knowshon & Denver Broncos & Running Back & 2013 & Unspecified (?) & Knee \\
\hline Nwaneri, Uche & Jacksonville Jaguars & Offensive Guard & 2013 & Unspecified (?) & Cartilage regrowth \\
\hline Owens, Terrell & Cincinnati Bengals & Wide Receiver & 2011 & $\begin{array}{l}\text { South Korea (Chaum } \\
\text { Anti-Aging Center) }\end{array}$ & $\mathrm{ACL}$ \\
\hline Rice, Sidney & Seattle Seahawks & Wide Receiver & 2013 & Switzerland (?) & Knee \\
\hline
\end{tabular}

Since 2009, 12 NFL athletes, including several high-profile players-Peyton Manning, Terrell Owens, and Ray Lewis-publicly acknowledged seeking unproven stem cell interventions for injuries sustained on the football field. Of the players who publicly identified the location or clinic they received treatment, half went to clinics outside of the United States. 
supporters argue that autologous cell therapies should be "treated as a medical practice" and left to the discretion and expertise of physicians [7]. The U.S. District Court disagreed, and later the Federal Appeals Court disagreed. As a result, these therapies must now be FDAapproved or performed at clinics outside the United States [8,9]. It is important to note that there is currently no standard definition of "minimal manipulation," and these court rulings only apply to this specific case and not all autologous SC therapies. However, the court ruling does provide a legal standing for the FDA to prosecute other clinics offering similar therapies.

The NFL players we identified, who sought therapies for pain or specific injuries, hailed from teams across the country (Table 1). While a few players acknowledged the clinic where they obtained the treatment, we found the majority of the information on blogs or news media outlets containing limited details. The discussion peaked in 2012 (Fig. 1). Several articles at this time were published in the mainstream media describing these kinds of therapies as the future standard of care [106-108]. These reports coincided with media reports of Peyton Manning, a very popular and highprofile player, traveling to Germany to obtain SCs for a football-related neck injury. Since then, public discussion of these treatments by professional athletes has declined. It is unclear whether the treatments themselves have declined in popularity or if government crackdowns on the clinics, including Regenexx and CellTex in the United States, limited player access. But this seems unlikely as there remain a multitude of clinics offering these treatments across the country. According to its website, the therapy by Regenexx alone is available at 23 clinics in 16 states, and Regenexx has conducted more than 2,000 SC procedures in the past 8 years [109].

Most clinics include a list of articles or references on their websites to validate their claims and treatments, but the majority of links consist of press releases,

FIG. 1. Years NFL players obtained stem cell treatments. The number of NFL players publicly known for receiving stem cell treatments peaked in 2012-the year that Indianapolis Colts' Peyton Manning (currently on the Denver Broncos) traveled to Germany to obtain stem cells for a football-related neck injury.

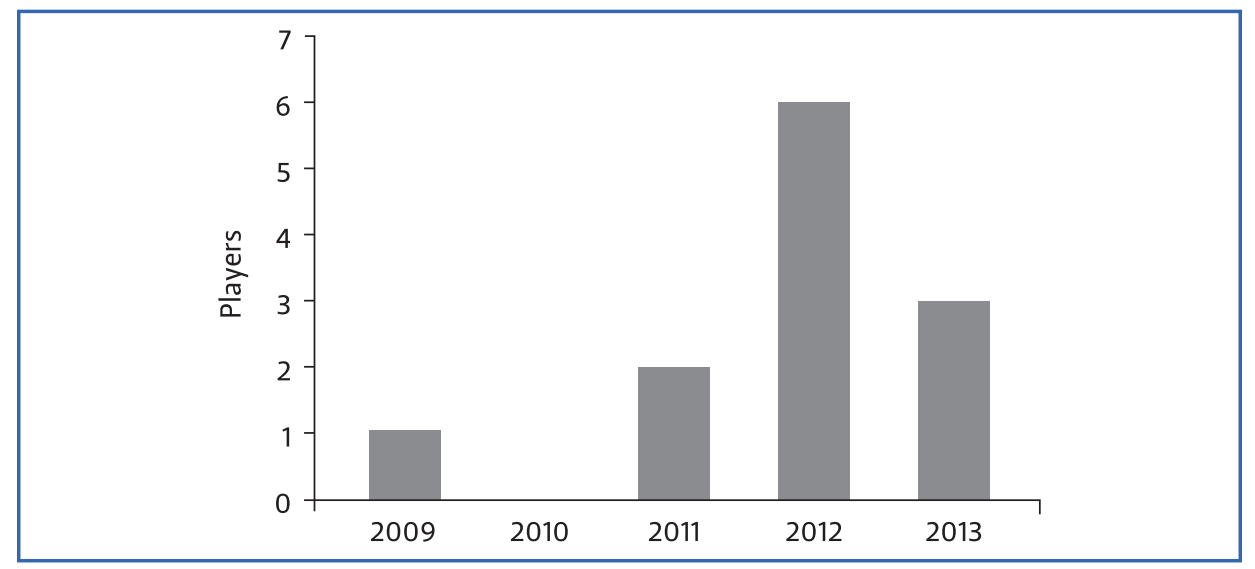

reviews, and news articles. The few peerreviewed articles only describe the safety of these procedures, the majority from authors linked to the clinics. For example, Regenexx cited 11 articles from authors working with their clinics prominently in the "Research" portion of the site. External research is buried in the "Frequently Asked Questions" section. The publications neither provided clinical data on dosage or effectiveness, nor showed FDA-approved clinical trial data. Furthermore, the procedures are not approved by the FDA or its equivalent in other countries. Since some of the therapies fail to meet FDA criteria, they must be conducted overseas. Without

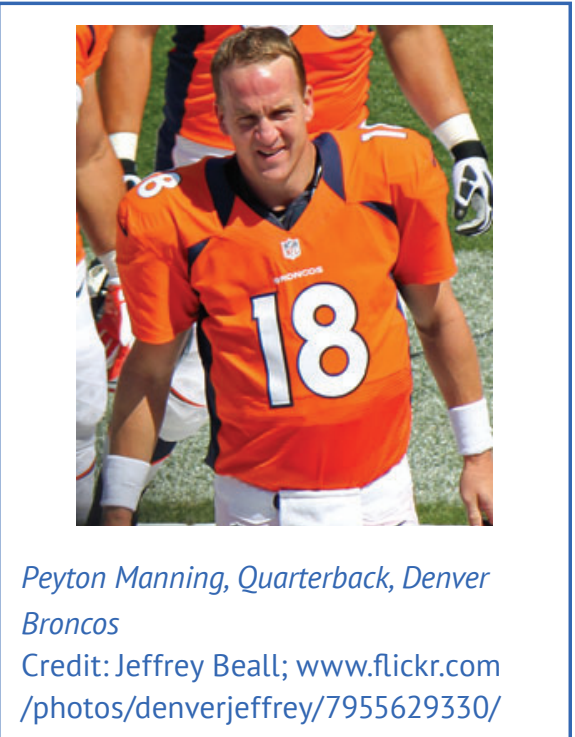

regulatory oversight and clinical data, potential patients do not have adequate information to understand the risks associated with the treatments and thus make uninformed decisions.

Several U.S.-based clinics now include athlete-targeted advertisements. One clinic, Precision Stem Cell, uses photos and quotes from Marquis Maze and Roland McClain for its procedure that is currently offered in Bogotá, Colombia [110]. Regenexx, a Colorado-based clinic, advertised that a player, Jarvis Green, sought treatment from them in 2010. However, the FDA soon thereafter filed an injunction against one of the three procedures Green obtained, so it is now carried out by Regenexx Cayman, "an independently owned and operated medical services provider" in the Cayman Islands [109]. SmartChoice Stem Cell Institute has a former NFL player, Tom McManus, as its official sponsor, although it is unclear if the player himself ever received SC treatments either at the clinic or at all [111]. In addition, multiple clinics use quotes from unnamed athletes and NFL players to advertise the validity of their therapies [112].

Furthermore, the use of NFL players to endorse these activities may lead the general public to view the procedures as safe and effective, even with a lack of scientific data to adequately defend the therapies. NFL players have outsized influence as celebrities 


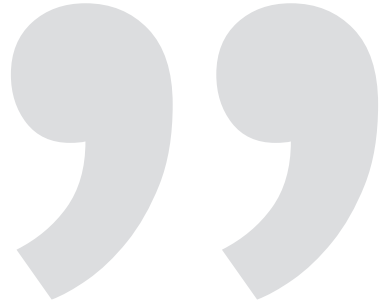

Use of NFL players to endorse these activities may lead the general public to view the procedures as safe and effective, even with a lack of scientific data.

and role models in U.S. society. Research has found that "famous athletes can have an important influence on the [public's] healthrelated knowledge, attitudes, and behavioral intentions" [10].

This relationship, as well as other forms of SC research aggrandizement, has the potential to aid in the expansion of the unregulated market of unproven SC clinics around the world [11]. Recent studies have demonstrated the impact of celebrities, including professional athletes, in the promotion of products from beer to tennis shoes [12-16]. Athletes have also been shown to be effective in raising awareness and changing behaviors related to health issues like HIV/AIDS and domestic violence [10]. The use of the athlete, a trusted figure, legitimizes unproven SC procedures and conveys a sense of safety and efficacy even when one does not exist. Further research should be conducted on the use of athletes in the promotion of these controversial clinical therapies to determine their impact and if they should be regulated by the government similarly to direct-to-consumer ads for pharmaceuticals.

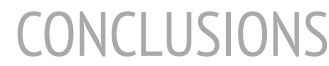

With the rise of new and unproven SC treatments, the NFL faces a daunting task of trying to better understand and regulate the use of these therapies in order to protect the health of its players. The online data on NFL players and the clinics where they obtained treatment suggest that players may be unaware of the risks they are taking. Furthermore, players who are official spokespersons for these clinics could influence others to view the therapies as safe and effective despite the lack of scientific research to support these claims.

The NFL and other sport leagues should review the procedures for SC treatment to determine how best to support, evaluate, and possibly regulate SC treatments to ensure the safety of their players and their followers. This could be organized similarly to the NFL investigations on the effects of concussions and traumatic brain injuries. After facing harsh criticism related to its handling of concussions, the NFL reshaped its controversial Mild Traumatic Brain Injuries Committee by naming two new chairmen, both renowned neural surgeons, and expanding the committee's efforts to include all head, neck, and spine injuries. The NFL also established new protocols for players who experience concussions during games and instated harsh penalties for not following these protocols. Furthermore, it began a $\$ 60$ million research effort called the Head Health Initiative and donated \$30 million to the National Institutes of Health for concussion research [17].

Using concussions as a model for approaching SC treatments, the NFL should convene an independent committee of medical professionals, without ties to the NFL or any of its teams, to evaluate the safety and efficacy of the therapies, establishing a set of criteria for what is considered "safe" and "effective." This committee would make recommendations to the NFL on unproven SC treatments and could also investigate other new and controversial medical interventions.

Furthermore, as with the study of concussions, the NFL should devote funds for research on SC therapies and evaluating the safety and success of previous studies. The NFL research could focus on safety of the treatment and the potential short- and long-term effects of the procedures. The research should also assess the claim that the therapies can shorten recovery times after injury and if the procedure should be considered "performance enhancing." Once they evaluate these items, the NFL, along with the NFL Players Association, can determine if and how they want to regulate $\mathrm{SC}$ treatments. By staying abreast of the development of these therapies, the NFL will protect itself and its players by enabling new and beneficial treatments while curbing illegitimate and unsafe usage.

\section{ACKNOWLEDGMENTS}

We thank George Romar and Monica Matsumoto for help with data and article preparation. This project is supported by the Baker Institute's Science and Technology Policy Program, as well as the Baker Institute's International Stem Cell Policy Program, which is endowed by the State of Qatar in collaboration with the Qatar Foundation.

\section{Author Disclosure Statement}

No competing financial interests exist.

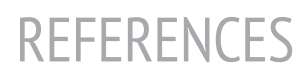

\section{Primary Literature}

1. Einsidel EF and H Adamson. (2012). Stem cell tourism and future stem cell tourists: policy and ethical implications. Dev World Bioeth 12:35-44.

2. Caulfield T and A McGuire. (2012). Athletes' use of unproven stem cell therapies: adding to inappropriate media hype? Mol Ther 20:1656-1658.

3. Lau D, U Ogbogu, B Taylor, T Stanfiniski, D Menon and T Caulfield. (2008). Stem cell clinics online: the directto-consumer portrayal of stem cell medicine. Cell Stem Cell 3:591-594. 
4. Zarzeczny A, C Rachul, M Nisbet and T Caulfield. (2010). Stem cell clinics in the news. Nat Biotechnol 28:1243-1246.

5. Matsumoto AM. (1990). Effects of chronic testosterone administration in normal men: safety and efficacy of high dosage testosterone and parallel dosedependent suppression of luteinizing hormone, follicle-stimulating hormone, and sperm production. J Clin Endocr Metab 70:282-287.

6. Lysaght $\mathrm{T}$ and AV Alastair. (2011). Regulating autologous adult stem cells: the FDA steps up. Cell Stem Cell 9:393-396.

7. Bianco P and D Sipp. (2014). Regulation: sell help not hope. Nature 510:336-337.

8. Cyranoski D. (2012). FDA's claims over stem cells upheld. Nature 488:14.

9. Musie M and I Hyun. (2014). A question of ethics: selling autologous stem cell therapies flaunt professional standards. Stem Cell Res. doi: 10.1016/j.scr .2014.04.014. [Epub ahead of print] 10. Brown WJ, MD Basil and MC Bocarnea. (2003). The influence of famous athletes on health beliefs and practices: Mark McGwire, child abuse prevention, and androstenedione. J Health Commun 8:41-57.

11. Caulfield T and C Condit. (2012). Science and the sources of hype. Public Health Genomics 15:209-217.

12. Lear KE, RC Runyan and WH Whitaker. (2009). Sports celebrity endorsements in retail products advertising. Int $\mathbf{J}$ Retail Distrib Manag 37:308-321.
13. Jones ME. (1979). Celebrity endorsements: a case for alarm and concern for the future. N Engl Rev 521:521-544.

14. Atkin C and M Block. (1983). Effectiveness of celebrity endorsers. J Advertising Res 23:57-61.

15. Choi SM and NJ Rifon. (2007). Who is the celebrity in advertising? Understanding dimensions of celebrity images. J Pop Cult 40:304-324.

16. Agrawal J and WA Kamakura. (1995). The economic worth of celebrity endorsers: an event study analysis. J Marketing 59:56-62.

17. Underwood E. (2013). NFL kicks off brain injury research effort. Science 339:1367.

\section{Websites}

101. Kiatpongsan S and D Sipp. (2008). Offshore stem cell treatments. Nat Rep Stem Cells. www.nature.com/stemcells/2 008/0812/081203/full/stemcells.2008.151 .html

102. ISSCR (2008). Guidelines for the Clinical Translation of Stem Cells. www .isscr.org/home/publications/ClinTrans Guide

103. Abbott A. (2011). Notorious stem cell therapy centre closes in Germany. Nature News Blog, 9 May. http://blogs.nature .com/news/2011/05/notorious_stem_cell therapy_ce_1.html

104. NFL Players Association: Banned Substances. www.nflplayers.com/About - us / Rules - Regulations / Player -Policies/Banned-Substances/
105. FDA: Code of Federal Regulations Title 21. www.accessdata.fda.gov/scripts /cdrh/cfdocs/cfefr/CFRSearch.cfm ?fr $=1271.15$

106. Assael S. (2011). Chasing the miracle cure: Peyton Manning won't be the last start linked to stem cell therapy. ESPN The Magazine. http://espn.go.com/espn/st ory/_id/7058209/peyton-manning-last -star-linked-stem-cell-therapy-espn -magazine

107. Franklin D. (2013). A dangerous game: some athletes risk untested stem cell treatments. Scientific America. www .scientificamerican.com/article/a -dangerous-game-athletes-risk-untested -stem-cell-treatments/?page $=1$ 108. Caulfield T. (2012). What does it mean when athletes get "stem cell therapy"? The Atlantic Monthly. www .theatlantic.com/health/archive/2012 /10/what-does-it-mean-when-athletes-get -stem-cell-therapy/263875/

109. Regenexx Website: www.regenexx .com/2012/10/update-from-former-nfl -player-jarvis-green-and-stem-cells/

110. Precision Stem Cell Website: www .precisionstemcell.com/

111. SmartChoice Stem Cell Institute Website: www.smartchoicestemcell. com/blog/2014/04/former-nfl-jaguar-to - be-the-s pokesperson-for-smart choice $\%$ C $2 \%$ AE-stem-cell-institute .aspx

112. Orthopedic Stem Cell Institute Website: http://orthopedicstemcellinstit ute.com/stem-cell-therapy-news-articles/ 